results from reinfection. This is caused by the prolonged excretion of the organism among untreated children, lasting up to seven weeks. ${ }^{5}$ On the other hand, stool cultures of all treated patients yielded negative results within 48 hours. ${ }^{5}$ Our patients indeed showed that simultaneous treatment is effective in preventing reinfection as it caused concomitant eradication of the infection in all the children.

It is concluded that in a nursery with multiple cases of campylobacter enteritis stool cultures should be taken from all children, including those who are asymptomatic at that time, because it is necessary to treat simultaneously all children whose cultures yield positive results. This approach is an effective method for eradicating the infection.

\footnotetext{
References

1 Blaser MJ, Reller LB. Campylobacter enteritis. N Engl J Med 1981;305:1444-52.

2 San Joaquin VH, Welch DF. Campylobacter enteritis: a 3-year experience. Clin Pediatr 1984;23:311-6.

${ }^{3}$ Blaser MJ, Waldman RJ, Barrett T, et al. Outbreaks of campylobacter enteritis in two extended families. J Pediatr 1981:98:254-7.

4 The child day care infectious disease study group. Public health considerations of infectious diseases in child day care centers. J Pediatr 1984;105:683-701.

5 Karmali MA, Fleming PC. Campylobacter enteritis in children. $J$ Pediatr 1979;94:527-33.

6 Walder M. The epidemiology of campylobacter enteritis. Scand J Infect Dis 1982;14:27-33.
}

Correspondence to Dr S Ashkenazi, Department of Pediatrics, Beilinson Medical Center, Petah Tiqva 49100, Israel.

Received 27 August 1986

\section{Commentary}

\section{H P LAMBERT}

\section{Department of Communicable Diseases, St George's Hospital Medical School, London}

It is often forgotten that antimicrobial drugs can sometimes be used to control the spread of gut infection. This 'epidemiological' use has a chequered history. Most often non-absorbable antibiotics, such as neomycin, have been employed to blanket an outbreak, especially of Escherichia coli gastroenteritis, and with variable results. Sometimes the outbreak was apparently controlled and at other times the attempt failed completely; certainly, antibiotic prophylaxis should not be used in this way unless combined with a rigorous attempt to tighten up normal hygienic methods of cross infection control.

This description of the control of campylobacter gastroenteritis in a day care centre makes a useful point. Features of this infection that perhaps make for a favourable outcome are:

(1) Erythromycin certainly seems to render stool cultures negative very quickly, usually within 48 hours of starting the drug (their references 1 and 6).

(2) The duration of carriage may be quite long without treatment, although $90 \%$ of patients do become negative spontaneously within two months.

(3) Campylobacter diarrhoea, although usually short lived, can be prolonged or recurrent, and it is patients with diarrhoea who pose most risk to their contacts. Although eight of the 12 contacts with positive stools were asymptomatic at the time of sampling, all of them had had recent diarrhoea.

\title{
Co-trimoxazole red cell aplasia in leukaemia
}

\author{
C E M UNTER AND G D ABBOTT
}

Department of Paediatrics, Christchurch Clinical School of Medicine, Christchurch Hospital, New Zealand.

SUMmary A 4 year old boy with acute lymphoblastic leukaemia developed a pure red cell aplasia 13 months after entering remission and while on maintenance chemotherapy. Co-trimoxazole was also being administered for prophylaxis against Pneumocystis carinii infection. When co-trimoxazole was stopped the red cell aplasia resolved.

Co-trimoxazole (sulphamethoxazole and trime- thoprim) provides effective prophylaxis against Pneumocystis carinii infection ${ }^{1}$ and is therefore commonly used during treatment for childhood acute lymphoblastic leukaemia. Haematological toxicity, particularly neutropenia and thrombocytopenia, has been described with co-trimoxazole, ${ }^{2} 3$ but selective aplasia of the bone marrow erythroid series is extremely rare and has not previously been reported in acute lymphoblastic leukaemia. We report a case of pure red cell aplasia that occurred in a child on maintenance treatment for acute lym- 